Témoigner Témoigner. Entre histoire et mémoire

Getuigen Revue pluridisciplinaire de la Fondation Auschwitz

118 | 2014

Au nom des victimes. Dictature et terreur d'État en Argentine, Chili et Uruguay

\title{
La plaque d'identité de David Jacob Zak
}

David Jacob Zak's identity plaque

Het identiteitsplaatje van David Jacob Zak

\section{Danielle Pinedo}

Traducteur : Philippe Mesnard

\section{(2) OpenEdition}

Journals

Édition électronique

URL : http://journals.openedition.org/temoigner/1231

DOI : 10.4000/temoigner.1231

ISSN : 2506-6390

Éditeur :

Éditions du Centre d'études et de documentation Mémoire d'Auschwitz, Éditions Kimé

Édition imprimée

Date de publication : 1 octobre 2014

Pagination : 21-22

ISBN : 978-2-84174-674-3

ISSN : 2031-4183

Référence électronique

Danielle Pinedo, "La plaque d'identité de David Jacob Zak », Témoigner. Entre histoire et mémoire [En ligne], 118 | 2014, mis en ligne le 01 octobre 2015, consulté le 23 octobre 2020. URL : http:// journals.openedition.org/temoigner/1231 ; DOI : https://doi.org/10.4000/temoigner.1231 


\section{LES FOSSES, LES FOUILLES ET LARCHÉOLOGUE}

TRACES Les fouilles de lieux de massacre ont empli le paysage mémoriel à partir des années 1990 et, parfois même, l'actualité de certaines contrées comme l'Espagne, 'Argentine, le Rwanda. A Sobibor, deux archéologues découvrent la plaque

d'identité d'un jeune Juif hollandais.

P t-être cela a-t-il vraiment émergé après les assacres qui ont eu lieu durant la guerre d'exwanda. On peut se rappeler des clichés que Gilles Peress a réalisés en 1996 des fosses communes aux alentours de Srebrenica, après que les populations locales ont éte assassinées par les troupes serbes Mlać M. Mladic. Mais on a aussilimpression que cetteprésence remuait nuis n'osait pas gratter la surface du présent de crainte qu'elle 1990, le phénomies années 1990, le phenomene gagne l'autres territoires, lEurope de . savait dejà. une terre gorgee de sang (emprunt au titre du livre de Timothy Snyder), où les tueries commises par les Sne (E) implement, ou cimple Père Desbois et son groupe ont mis encifs. Ce que le Père Desbois et son groupe ont mis en œuvre a técien de la Baltique à la mer Noire.
Mais aux autres bouts du monde (de l'Argentine et du Chili jusqu'au Guatemala de l'Algérieà l’Afrique du du Chil...jusquau Guatemala, de I'Algerie a l'Afrique du Sud, du Canbodge à l'on lon pasa à sodiquedo sodquement, provisoirement, lacruauté pratiquée toute inpunite par les titire nation einsi ainsisusceptibledecontenir - sombre civite, mais a des crim non descrimes commis juste apres celle-ci, quand le Franquisme nettoyait le pays de ses opposants. Toutes ce lations de morts sans sépulture qui, pont les popu-

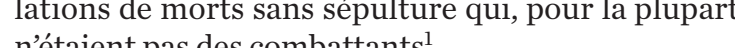

Comme le fait très justement remarquer Annette Becker dans ce même numéro, les combattants s'enterrent, on donne même une tombe à l'ennemi alors que les criminels essaient généralement de faire disparaître non seulement les corps des victimes quils ont massacrés, quils ent uilises pou noys dispant une C's pour les faire disparatre. Cest alors qu'un nouvel actenr de la mênoire apparait de façon très discrète, quasing tes années 1990. Il ai tournant des ances 19. nl vient de ce qui - pour nous, immergés dan ous l'espèce de l'immémorial l'án Car une partie de cette histoire dontles ruines sogue. Car une partie decette histoire dont les ruines sont les estes inprobables des corps revient à nous pour faire chaque parle des lieux immonde

Or, tout à fait par hasard, nous retrouvons dans le quzureketYohä d’archéologues dont nous avions déjà précédemment publié un texte coécrit avec leuréminent collègue Isaac Gilead $^{2}$. Il y est question de la plaque retrouvée d'un enfant juif hollandais déporté à Sobibor. C'est l'article sur cette découverte dont nous livrons la traduction ci-dessous. I
(1) Cf. l'exemple de ces cinq femmes assassinées le 26 septembre 1936 "Fosses communes, paysages de la ta terreur " , in Témoigner entre histoire
Foster némoire, $n^{\circ} 115, \mathrm{p} .44-67$ ).

(2) Isaac Gilead, Yoram Haimi, Wojciech Mazurek, «Excavating Nazi décembre 2012 entres 1, in Témoigner entre histoire et mémoire, ${ }^{\circ} 11$

\section{La plaque d'identité de David Facob zak}

Par Danielle Pinedo. Titre original : « $\mathrm{De}$ jongen die voortleeft door dit naamplaatje
NRC Handelsblad, mercredi 12 juin 2013

À Sobibor moururent des milliers de Juifs néerlandais. Des archéologues trouvent une plaque d'identité au nom de David Jacob Zak: " on a l'impression que son âme continue à vivre.

avid Jacob Zak, plus connu sous le diminutif de « Dedàl'image stéréotypée du jeune garare des cheveux blonds quil peignit vec une raie sur le côté. Durt la guerre, il aurait pu se cacher facilement $"$, déclare sa cousine ies Caransa aujourd'hui âgé de 74 ans. "Grâcè̀ jon physique, personne n'urait faitattention à Jusqu’à la moitié de la guerre, David habitait avec ses parts Uitwaardenstrat à Amsterdam. Son père était diamantaire. mère femme au foyer. Sur une photo datant de 1939, David se trouve entre ses parents, bien

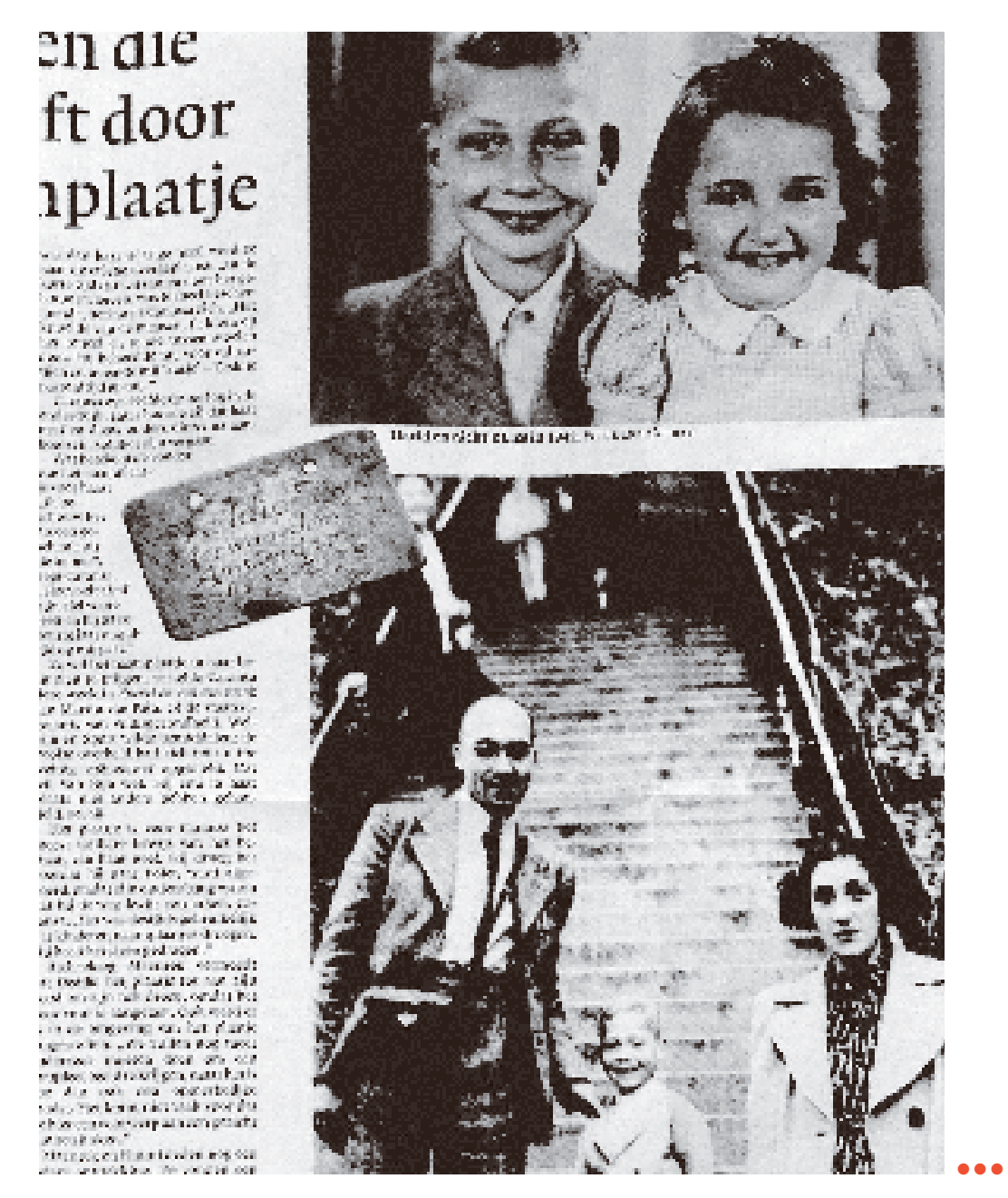


.. campé sur ses jambes, en bas d'un escalier. Son père, Simon, le tient par lepaule et sa mere, Judith, rsurée.

Le week-end dernier [8-9 juin 2013], on apprend que deu placed'identité au nom dé d'exterde Ded de Sobibor con a Sobibor en Pologne. Ces deux archeses, un Polonais et un Israen, faisaient des recherches in enes ubjet metallicue. « Cest une plaque artisanale avec des lettres

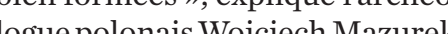
Ce depor Ce dernier et son collegue, Yoram luann, essaient de reconstituer

Ja troutes sobibor.

La trouvaille est exceptionnelle, car peu dobjets personnel Après l'insurrection du 14 octobre 1943 pendentlaquelle prisonniers ont fui le camonsoprisonicis ont fui lecanp, quasint toutes les traces de lextermarion nazie ont été élacées. Par Judith de la Penha une jeune flle d'Am fait une recons the Léa avait juste six ans quand elle été gat jén six ans quand elle a été gazée », explique Wojciec

David et ses parents partiren 6 juin 1943 avec un transport Cenfants de Wecterbork. Selon ćté rensá, cousine de David, ils ont pour être conduit au Hollandsche pour êtue zondita an Hollandsche Schoumburg, lép de rassenblement pour la deportation des Juif non loin du parc zoologique dArdiriós vers le camp de Vught ${ }^{3}$ oü
Is ont séjourné un mois. Caransa, avril, arriva au Holqu'elle était de quatre ans plus quelle était de quatre ans plus jeune que son cousin, qui en avait Pentea té placée danslacreche. pendant ce cours mont, nous lavait une attitude protectrice a mon égard $x$, se souvient-elle. $C$ mon ègard ", se souvient-elle. Ça ur la scène et courrait entre les haises "Il memper chat do tom" Deddie me protén "pette sour" Carana surécut à la guere étant carvecut à la guerre étant cachée. Pus tard, ell prientété gás dès lespar. à Sobibor Que signifie la découà Sobibor. Que signifie la découJe de cette plaque pour elle ? "Je considere que cest conne un cadeau du ciel», dit-elle. « Jai le à vivine que son ane continu à vise et quau bout de soixante.ix ans, il me protege toujours. ion de l'objet, déclare-t-elle lor de l'émission, Brienter Brink de Mán van Rjh. Or, sile santé du bien-être et des sports f érlandais est tout à fait dispose

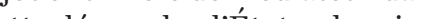
de son côté une attitude peu coo-

La plaque est pour Carensa l preuve la plus tangible de tence de son cousin. Il la portait part sa derportation en Pologne, qu'il ques en che quil se perde enchenin. « Cectait courant à lepoque que les enfants pertent a ter por-elle.

Wojciech Mazureksuppose que Deddie porta sa plaque autour du cou jusqưà sa mort, parce qưelle a été abîmée et que l'on a également de sa plaque On devra faire de recro image complète de ce qui s' passé, mais de toute ço qui s'est passé, mais de toute façon cest une rive sions mo sions mettre un visage sur un objet. fait une autre découv vèrentur possible qu'il aitété creué por détenus qui devaie processus d'extermination égacessus d'exteŕnination. «On a six squelettes dans ossements de cix sque te un tube dentevre néerlandais et Mazurek Seto la Mazurek. Selonlarchéologue, liy Tade deup à Pll

Tous nos remerciements à la rédaction
de $N R C$ pour leur autorisation de reproduction

(1) Le chiffre des Juifs qui ont pu sévader reste approximatif, quelques centaines, trois cents
environ car la plupart n'a pas survécu à la guerre. Néanmoins, après-guerre, c'est une
quarantaine de rescapés qui ont témoinné quarantaine de rescapés qui ont témoigné
(cf. Sila Cehreli, Témoignage du Khurbn. La résistance juive dans les centres de mise à mort
- Chelmno, Belzze, Sobibor, Treblinka, Paris,
Kinâs (2) Situé au nord-est des Pays-Bas, le camp
de Westerbork était le principal camp de rassemblement des Juifs hollandais avant leur déportation, équivalent de Drancy pour la

(3) Un des camps de concentration sous
contrôle de la SS dans le sud des Pays-Bas, non loin de Bois-le-Duc et proche de la Belgique. Sur les 31000 détenus, 15000 Juifs
y ont séjouné. Les 5 et 6 jun 1943 tous les enfants du camp ont été déportés, via Westerbork, à Sobibor où ils ont tous
été assassines.

(4) Tublik.nl/knevel-van-den-Brink/10-
juin-2013 